\title{
Microbiology Susceptibility Link Identifier
}

National Cancer Institute

\section{Source}

National Cancer Institute. Microbiology Susceptibility Link Identifier. NCI Thesaurus. Code C162019.

A sequence of characters used as a linkage between related microbiology susceptibility test identifier records. 